\title{
RELATION BETWEEN STATIONARY AND MOVING PEDESTRIAN LOAD MODELS
}

\author{
JAN ŠTĚPÁNEK*， JiŘí MÁCA \\ Czech Technical University in Prague, Faculty of Civil Engineering, Thákurova 7, 16629 Prague 6, Czech \\ Republic \\ * corresponding author: jan.stepanek@fsv.cvut.cz
}

\begin{abstract}
The dynamic analysis of footbridges became common in recent years, but the approach to modelling pedestrian induced load is still inconsistent. This paper compares two different ways of modelling single pedestrian load. The response of the structure to stationary pulsating force is compared to the results given by the same force moving along simply supported beam. 300 randomly generated beams were used in this parametric study to describe the relation among these two approaches and parameters of the structure. The proposed formula was successfully applied to get more realistic estimation of response of the structure to a single walking pedestrian using stationary pulsating force.
\end{abstract}

KEYWORDS: Footbridges, pedestrian load models, forced vibration, response of structure.

\section{INTRODUCTION}

Footbridges designed and built during recent years are very slender and flexible structures in many cases. Such footbridges may be very susceptible to pedestrian induced load which may cause an uncomfortable level of vibration. Natural frequencies of such structures are often very close to walking frequency of pedestrians, and therefore the resonance effect may occur.

A number of studies have been made in past decades to define the procedure of effective and safe design of footbridges. A footbridge designed this way has to satisfy the criteria of serviceability limit state (SLS). To be able to detect the risk of unacceptable vibration during design phase the dynamic analysis is often necessary. The European codes define the maximal level of acceleration of a bridge deck which can be reached to keep the sufficient comfort of pedestrians. Numerical simulation can reveal the sensitivity of the structure due to pedestrians but unfortunately there is still a lack of pedestrian load models in current Eurocodes.

Many single pedestrian load models have been developed [1, 2] but most of them assume the numerical analysis using moving forces or moving biodynamic models. Nevertheless, most of the commercial programs used for static design of bridges do not provide the option of calculation with force moving along the structure.

Stationary harmonic pulsating force was presented in guidebook [3] to simulate single walking pedestrian. The advantage of this estimation is its simplicity when it comes to numeric analysis in static software. The main disadvantage of this load model is that it does not consider the time that one spends on the bridge. The stationary pulsating force without time limitation may cause overestimation of the response of the structure which leads to unnecessary design changes and potential increase of cost of the structure.
This paper focuses on comparison of approaches of modelling single pedestrian load by stationary and moving pulsating force. The goal is to find a simply applicable formula which combines these two approaches. The formula can be used to find realistic estimation of response of a simply supported beam using stationary pulsating force.

\section{Pedestrian loAd MOdels}

Pedestrian load can be divided into several categories. Normal traffic on the bridge can be defined as a single pedestrian load, group of walking people or stream of pedestrians. Exceptional load situations (jumping, running, kneeling or vandalism) must not excite the intolerable vibrations either [2]. To be able to evaluate the response of the structure in normal operation, it is necessary to define a simple but accurate dynamic single pedestrian load model.

\subsection{Single PEDESTRIAN LOAD}

Dynamic force produced by a single person can be represented by three components in perpendicular directions: vertical, horizontal lateral and horizontal longitudinal components [1]. Horizontal longitudinal force is usually considered negligible. The lateral component is caused by moving centre of gravity horizontally while walking. The frequency of such force is half than the stride frequency. A vertical component with the stride frequency is the cause of most of the documented problems with uncomfortable vibrations of footbridges. Only the vertical component is further investigated in this paper.

Vertical pedestrian load, assuming to be perfectly periodical force, can be formulated by Fourier series in Equation (1). Numerous different approximations of such formulation were developed in the past years [1, 4. 


$$
F_{v}(t)=G+G \sum_{i=1}^{n} \alpha_{i} \sin \left(2 \pi i f_{p} t+\varphi_{i}\right)
$$

where:

$$
\begin{array}{ll}
F_{v}(t) & \text { - vertical pedestrian load } \\
G & \text { - load corresponding to pedestrian's weight } \\
\alpha_{i} & \text { - Fourier coefficient of the respective member } \\
f_{p} & \text { - step frequency } \\
\varphi_{i} & \text { - phase shift } \\
n & \text { - total number of included members }
\end{array}
$$

Various coefficients $\alpha_{i}$ and $\varphi_{i}$ can be found in literature [1, 4, 5]. In general, it can be said that including more than three or four harmonic members has no effect on the final load due to low values of $\alpha_{i}$ for higher components.

The worst-case scenario, when the stride frequency is equal to one of the natural frequencies of the bridge, is usually adopted for simulating a single pedestrian walking along the structure [1, 4, 6. Pedestrian walking velocity $v_{s}$ also plays an important role in serviceability verification of the structure. The speed $v_{s}$ can be taken by Eq. 2

$$
v_{s}=f_{s} l_{s}
$$

Where $f_{s}$ is step frequency and $l_{s}$ is step length. The recommended mean value for $l_{s}$ also varies in different studies. Guidebook [4 recommends $l_{s}=$ $0.9 \mathrm{~m}$. According to guidebook [3] $l_{s}$ is dependent on step frequency and for $2 \mathrm{~Hz}$ (defined as normal walk) the typical step length $l_{s}=0.75 \mathrm{~m}$ is considered.

\subsection{StATIONARY PEDESTRIAN LOAD MODEL}

The stationary pulsating force can be a simple way to find out the response of structure to dynamic pedestrian load. The guidebook [6] recommends to apply stationary harmonic force with amplitude $280 \mathrm{~N}$ to the most adverse position of the structure. The value of the force is taken as $700 \times 0.4=280 \mathrm{~N}$ which is in accordance with average pedestrian weight $70 \mathrm{~kg}$ and the first Fourier coefficient 0.4. Other approaches take the movement of a person in account. The amplitude of applied force is multiplied by the reduction constant $\mathrm{R}$. Allen And Murray give $\mathrm{R}=0.7$ while Grundman takes $\mathrm{R}=0.6[1$.

The formula for reasonable reduction factor $\mathrm{R}$ for simply supported beam is set in further parametric study.

\subsection{MOVING PEDESTRIAN LOAD MODEL}

The moving load models can be used to define the response of the structure to pedestrian load in a very realistic way [5, 7]. Examples of more sophisticated load models, including biodynamic models and inverted pendulum can be found in 8. Many force models are summarized in [1, 4, and most of them contains first Fourier coefficient $\alpha_{1} \approx 0.4$. For this reason, one of the simplest models proposed by Charles and Hooprah was used in this study to simulate a walking pedestrian. This model consists only of static force $G$ and first harmonic member for $\alpha_{1}=0.4$. This simplified approach is also tolerated in guidebook [2] for simulation of a walking pedestrian.

\section{Parametric Study}

In this chapter a numerical analysis of 300 simply supported beams with randomly generated parameters was performed to find the formula for the reduction factor R. Both stationary a moving pulsating force were used to find the peak acceleration in vertical direction. Value of $\mathrm{R}$ defined in Eq. (3) was taken for every single structure. Finally, the function of $\mathrm{R}$ was found using the least-squares approximation with parameters of the tested beams used as the basis functions.

$$
R_{c a l}=\frac{a_{m o v}}{a_{s t}}
$$

Where $a_{m o v}$ is peak acceleration all over the structure calculated using moving force model and $a_{s t}$ is acceleration induced by stationary force model without time limitation.

\subsection{SET OF SIMPLY SUPPORTED BEAMS}

All the tested structures share the scheme of simply supported beam. Every beam was generated so that one of the three lowest natural frequencies equals to $2 \mathrm{~Hz}$. The i-th natural frequency of simply supported beam can be calculated from analytical equation (4).

$$
\omega_{i}=\pi^{2} i^{2} \sqrt{\frac{E I_{y}}{\mu l^{4}}}
$$

Where:

$$
\begin{array}{ll}
\omega_{i} & \text { - i-th natural circular frequency } \\
E & \text { - modulus of elasticity } \\
I_{y} & \text { - cross sectional second moment of area } \\
\mu & \text { - mass of one meter of length } \\
l & \text { - span length }
\end{array}
$$

$I_{y}$ can be expressed:

$$
I_{y}=\frac{\mu l^{4} \omega_{i}^{2}}{\pi^{4} i^{4} E}
$$

and $\mu$ is defined in Eq. (6).

$$
\mu=\rho A+\mu_{a d}
$$

If $\omega_{i}=2 \pi \times 2 \mathrm{~Hz}$, every other parameter excluding $I_{y}$ can be randomly generated and $I_{y}$ is found using Eq. (5). This procedure allows to generate simply supported beams with i-th natural frequency equals to $2 \mathrm{~Hz}$.

Assuming all the theoretical structures have rectangular cross-section, its width $b$ and height $h$ can be found by solving system of two equations (7) and (8).

$$
A=b h
$$




$$
I_{y}=\frac{b h^{3}}{12}
$$

Where:

$$
\begin{array}{ll}
A & \text { - cross sectional area } \\
\rho & \text { - material density } \\
\mu_{a d} & \text { - mass added on each meter of length without } \\
& \text { static effect }
\end{array}
$$

A single material - reinforced concrete C 30/37 was chosen for all the theoretical beams.

The ranges of randomly generated parameters:

$\rho=2500 \mathrm{~kg} / \mathrm{m}^{3}$ - standard density of concrete C $30 / 37$

$E=32 \mathrm{GPa}$ - modulus of elasticity of concrete C30/37

$\omega_{i} \quad=12.5664-$ circular frequency for $f_{i}=2 \mathrm{~Hz}$.

$l \quad=10-50 \mathrm{~m}-$ The typical span length of footbridges with static scheme of simply supported beam.

$A=0.03-3 \mathrm{~m}^{2}$ - Wide range of cross sectional area which allows sufficient diversity of structures in the set.

$\mu_{a d}=0-300 \mathrm{~kg} / \mathrm{m}$

$i \quad=1-3-$ One of the first three natural frequencies equals to $2 \mathrm{~Hz}$

$\xi \quad=0.008-0.02$ - damping ratio of concrete footbridges 1

The goal of this chapter is to assemble a set of simply supported beams with wide range of parameters so that the statistical analysis can be done using the leas-squares method. It means that some of the structures could exist just as mathematical model, not a real structure, because of their slenderness or high susceptibility to vibration. Some other conditions of the properties of generated beams are set to reduce the amount of such structures in the list:

- $\mathrm{h}>0.1 \mathrm{~m}, \mathrm{~b}>0.1 \mathrm{~m}$ - minimal height and width of the cross section

- $0.1<\mathrm{h} / \mathrm{b}<10$ - minimal and maximal ratio of height and width

- $\mathrm{h} / \mathrm{l}<0.1$ - maximal ratio of cross sectional height and span length

- $\mathrm{b} / \mathrm{l}<0.5$ - maximal ratio of width and span length

The final set of the structures is composed of three parts. Each part contains 100 beams with i-th natural frequency $2 \mathrm{~Hz}$ for $\mathrm{i}=1-3$. Every structure is modelled using finite element method (FEM) in MATLAB and consists of 50 beam elements. The shear deformation of elements is neglected according to Bernoulli-Navier hypothesis. This simplification can be applied thanks to condition $\mathrm{h} / \mathrm{l}<0.1$.

\subsection{Response of the StRUCture}

\subsubsection{RESPONSE TO STATIONARY PULSATING FORCE}

The i-th mode shape with corresponding natural frequency $2 \mathrm{~Hz}$ must be found at first. The vertical harmonic force $F_{v}(t)$ with amplitude of $280 \mathrm{~N}$ and frequency $f_{v}=2 \mathrm{~Hz}(9)$ is then applied in the most adverse position of the bridge.

$$
F_{v}(t)=280 \sin \left(2 \pi f_{v} t\right) \quad \mathrm{N}
$$

Maximal displacement of the structure is found using Newmark- $\beta$ method for solving equation of motion:

$$
K r(t)+C \dot{r}(t)+M \ddot{r}(t)=p(t)
$$

Where:

$$
\begin{array}{ll}
K & \text { - stiffness matrix } \\
C & \text { - damping matrix } \\
M & \text { - mass matrix } \\
r(t) & \text { - vector of displacement } \\
\dot{r}(t) & \text { - vector of velocity } \\
\ddot{r}(t) & \text { - vector of acceleration } \\
p(t) & \text { - load vector created from } F_{v}(t)
\end{array}
$$

Maximal acceleration can also be found solving the system of $2 \mathrm{~N}$ linear equations (11) for system of $\mathrm{N}$ degrees of freedom. This approach is technically much easier but it does not provide any information about time history.

$$
\left[\begin{array}{cc}
\left(K-\omega^{2} M\right) & -\omega C \\
\omega C & \left(K-\omega^{2} M\right)
\end{array}\right]\left\{\begin{array}{l}
r_{s} \\
r_{c}
\end{array}\right\}=\left\{\begin{array}{l}
f_{s} \\
f_{c}
\end{array}\right\}
$$

Where $\omega$ is the circular frequency of pacing force and $r(t)$ and $p(t)$ are expressed by two components:

$$
\begin{aligned}
& r(t)=r_{s} \sin (\omega t)+r_{c} \cos (\omega t) \\
& p(t)=p_{s} \sin (\omega t)+p_{c} \cos (\omega t)
\end{aligned}
$$

The amplitude of acceleration $\ddot{r}$ of such harmonic motion is given by Eq. (14).

$$
\ddot{r}=\omega^{2} \sqrt{r_{s}^{2}+r_{c}^{2}}
$$

The reference value $a_{s t}$ is finally found as maximal value of acceleration vector $\ddot{r}$ in vertical direction.

\subsubsection{Response to MOVING PUlsating FORCE}

The pedestrian load model, used in this section, was adopted from [1]. It consists only of static weight of pedestrian $G=70 \mathrm{~kg}$ and the first member of Fourier series $\alpha_{1}=0.4$ and is expressed by Eq. (15). The model is moving along the structure. To define the velocity of its movement, the step length needs to be defined. The step length $l_{s}=0.75 \mathrm{~m}$ which is appropriate for step frequency was adopted from [3]. The walking speed was set according to Eq. (2). 


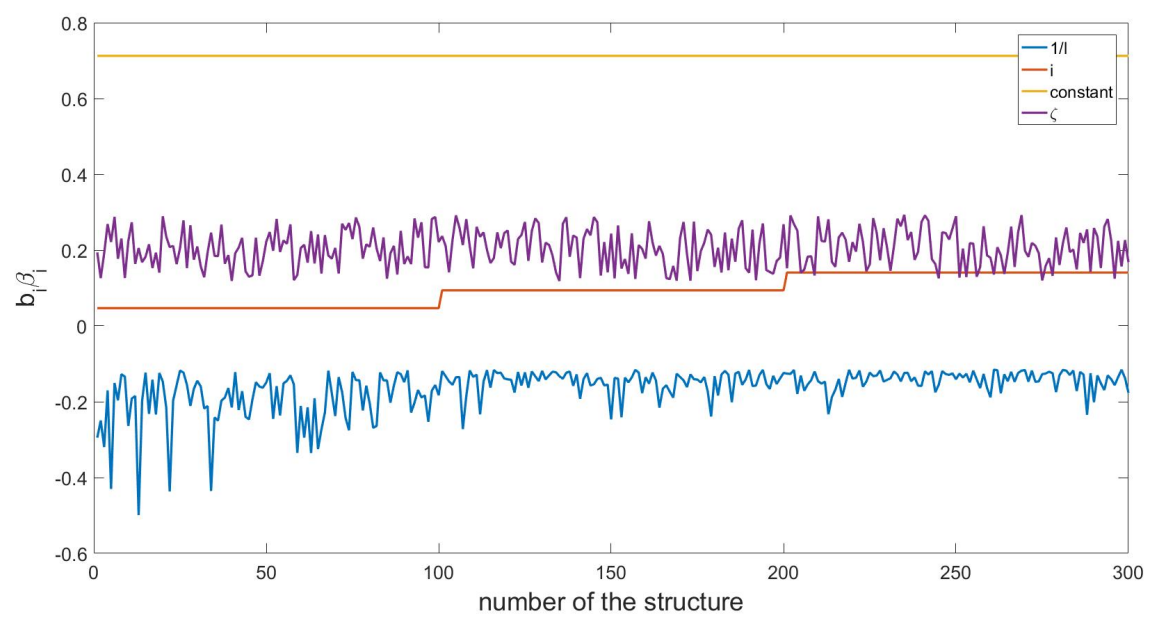

FIGURE 1. Separate contributions of basis functions.

$$
F_{v}(t)=700+280 \sin \left(2 \pi f_{v} t\right) \quad \mathrm{N}
$$

The peak acceleration $a_{m o v}$ in vertical direction reached on each structure was analysed using Newmark- $\beta$ method.

\subsection{Evaluation}

The peak accelerations $a_{s t}$ induced by stationary force model and $a_{m o v}$ induced by the moving force model were evaluated for all the structures which resulted in 300 values of reduction coefficient $R_{\text {cal }}$ Eq. (3)

The least-squares method was used for determination of the relation between reduction coefficient $R$ and the parameters of the structure. Eleven basis functions $b_{j}$ were defined:

(1.) $l$ - span length

(2.) $\mu$ - mass for 1 meter of the beam

(3.) $I_{y}$ - cross sectional second moment of area

(4.) $\mu l$ - total mass of the structure

(5.) $\frac{1}{l}$ - multiplicative inverse of span length

(6.) $\frac{1}{\mu}$ - multiplicative inverse of mass for 1 meter of the beam

(7.) $\frac{1}{I_{y}}$ - multiplicative inverse of cross sectional second moment of area

(8.) $i$ - the order of frequency which equals to $2 \mathrm{~Hz}$.

(9.) constant

(10.) $\xi_{1}$ - damping ratio of the first mode shape

(11.) $\frac{1}{\xi_{1}}$ - multiplicative inverse of damping ratio of the first frequency

The coefficients $\beta_{j}$ of basis functions were found solving system of equations 16 .

$$
\left[\begin{array}{cccc}
X_{11} & X_{12} & \cdots & X_{1 n} \\
X_{21} & X_{22} & \cdots & X_{2 n} \\
\cdots & \cdots & \ddots & \vdots \\
X_{m 1} & X_{m 2} & \cdots & X_{m n}
\end{array}\right]\left\{\begin{array}{c}
\beta_{1} \\
\beta_{2} \\
\vdots \\
\beta_{n}
\end{array}\right\}=\left\{\begin{array}{c}
y_{1} \\
y_{2} \\
\vdots \\
y_{m}
\end{array}\right\}
$$

Where $X_{i j}=\sum_{k=1}^{300} b_{i, k} b_{j, k}$ and $y_{i}=\sum_{k=1}^{300} b_{i, k} R_{c a l, k}$ and $k$ stands for number of the structure in the list.

The first calculation was primarily performed in order to define the parameters which have the greatest influence on the value of reduction factor. Four functions (no. 5,8,9 and 10) were chosen for further analysis.

The second calculation was performed using only four selected functions and the respective coefficients $\beta$ were found.

The function $R_{\text {cal }}$ can be approximated by $R_{a p p}$ which is expressed as a linear combination of basis functions (17). The Figure 1 shows the contributions of chosen basis functions.

$$
R_{c a l} \approx R_{a p p}=\sum b_{j} \beta_{j}
$$

The formula for $\mathrm{R}$ is finally set by equation:

$$
R=\frac{-6.248}{l}+0.0495 i+14.42 \xi_{1}+0.726
$$

and is usable in reduction of the stationary pulsating force model of single walking pedestrian according to Eq. 19.

$$
F_{v}(t)=R 280 \sin \left(2 \pi f_{v} t\right) \quad \mathrm{N}
$$

As can be seen in Figure 2, all the values of $R_{\text {cal }}$ falls in range between $0.5-1$ thus the range of meaningful values is defined as:

$$
0.5 \leq R \leq 1
$$




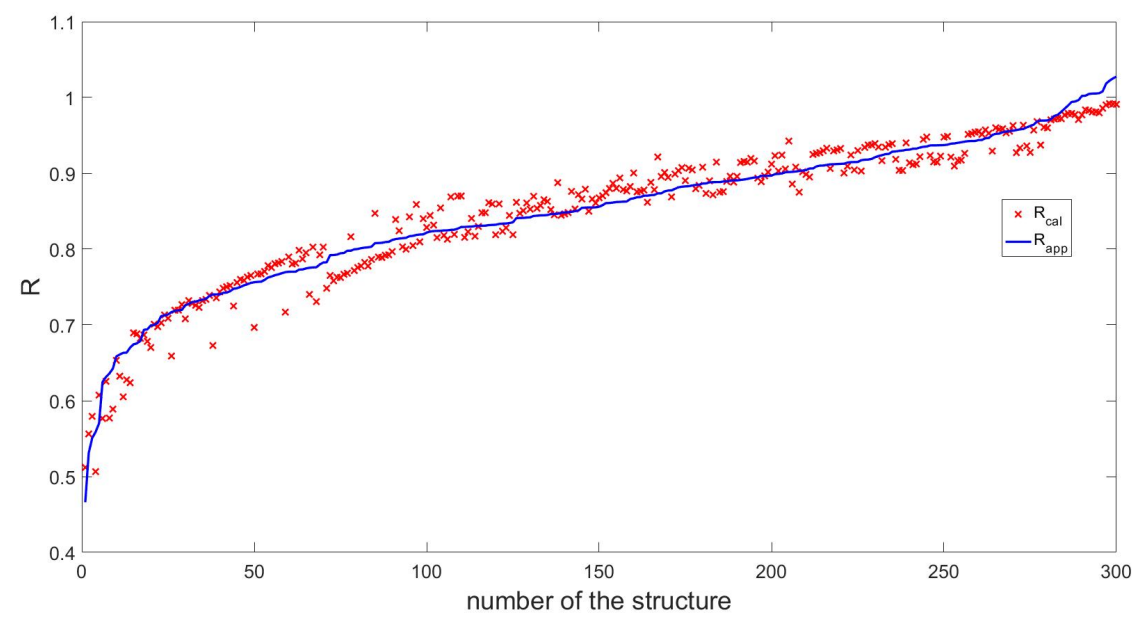

FiguRE 2. Calculated and approximated values of $\mathrm{R}$.

The average relative error of the approximation $\Delta R$ calculated according to Eq. 21) is equal to $2.3 \%$. Taking all the uncertainties of pedestrian load models into account, the value of relative error is sufficiently small.

$$
\Delta R=\left|\frac{R_{c a l}-R_{a p p}}{R_{c a l}}\right|
$$

\section{VERIFICATION OF THE PROPOSED FORMULA}

Verification of the proposed formula has been performed on numerical model of experimental structure presented in [5]. The experimental footbridge is prestressed concrete beam with $\mathrm{T}$ cross section and short overhangs (Figure 5). The tuned mass damper (TMD) was installed under the midspan of the beam to show its influence on the susceptibility to vibration. TMD allows the options to be locked, which is performed by stiff connection to the structure.

The moving load model used in section 3 provided very accurate results of response of the structure in comparison to load tests [5].

Calculations were performed with both locked and free TMD to verify the formula for reduction factor $\mathrm{R}$ (18).

The first natural frequency of the structure $f_{1}=$ $1.98 \mathrm{~Hz}$, and therefore the step length of $0.75 \mathrm{~m}$ can be applied according to guidebook [3]. Several parameters of the structure are required to estimate the reduction factor $\mathrm{R}$ :

- $\mathrm{l}=19 \mathrm{~m}$

- $\mathrm{i}=1$ (the first natural frequency is close to $2 \mathrm{~Hz}$ )

- $\xi_{1}$, damping ratio of the first mode shape $=0.0143$ (locked TMD), 0.06 (free TMD)

\subsection{LOCKED TMD}

The peak accelerations at midspan $a_{\text {mov }}$ using moving pedestrian load model according to Eq. 19 was

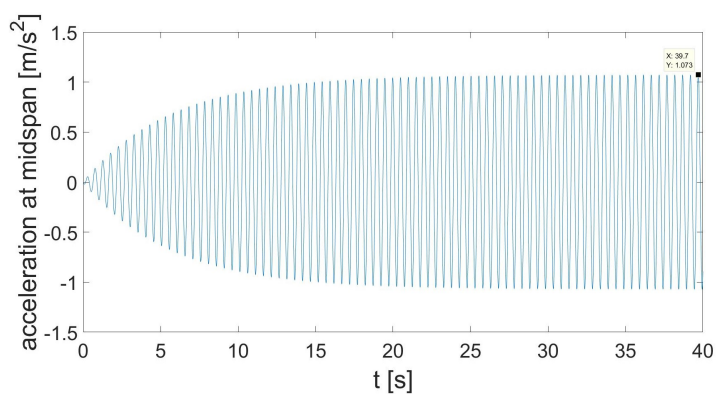

Figure 3. Response of the structure - stationary pedestrian load, locked TMD.

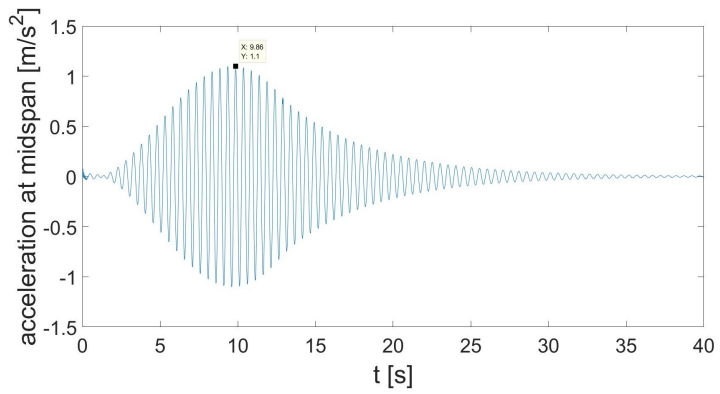

Figure 4. Response of the structure - moving pedestrian load, locked TMD.

evaluated and can be seen in Figure 4 . The value for reduction coefficient was calculated from Eq. (18).

Calculation of $\mathrm{R}$ for locked TMD:

$$
\begin{aligned}
R & =\frac{-6.248}{l}+0.0495 i+14.42 \xi_{1}+0.726 \\
R & =\frac{-6.248}{17.4}+0.0495 \times 1+14.42 \times 0.0143+0.726 \\
R & =0.623
\end{aligned}
$$

The amplitude of the stationary pulsating force is then $R \times 280=174 \mathrm{~N}$. Applying this force according to Eq. 19 in the midspan of the beam, the maximal acceleration $a_{s t}$ was evaluated. 


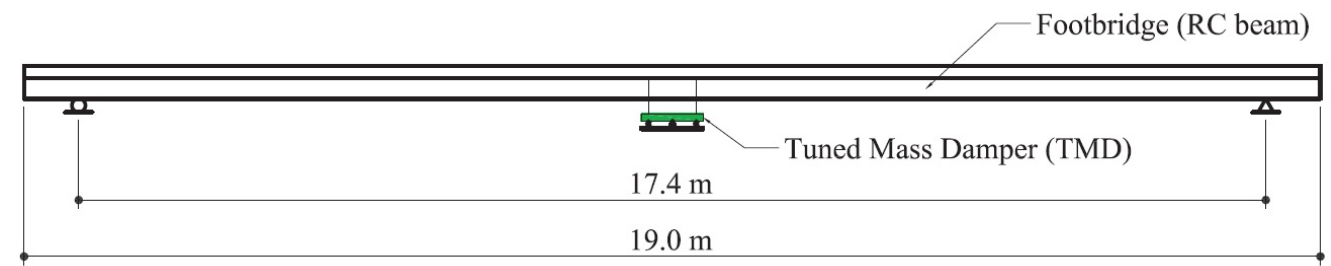

FiguRE 5. Static scheme of experimental footbridge [5].

\begin{tabular}{lccc}
\hline TMD & $\mathrm{R}$ & acceleration - moving model $\left[\mathrm{ms}^{-2}\right]$ & acceleration - stationary model $\left[\mathrm{ms}^{-2}\right]$ \\
\hline locked & 0.623 & 1.100 & 1.073 \\
free & 1 & 0.264 & 0.261 \\
\hline
\end{tabular}

TABLE 1. Maximal acceleration at midspan.

\subsection{FREE TMD}

The evaluation of acceleration with free TMD was performed using the same method as with locked TMD. Due to high structural damping $(\approx 6 \%)$, the reduction factor $\mathrm{R}$ exceeded limit value 1 which was presented in Eq. 20). Therefore, the value of $\mathrm{R}$ is set to 1 . Comparison of the results can be seen in Table 1

\section{Conclusions}

The formula for the reduction coefficient $\mathrm{R}$, which can be used for simulation of single pedestrian load, was proposed. This formula simplifies the pedestrian load from moving force model to stationary harmonic force model but keeps the accuracy of moving model. The formula is applicable on footbridges with static scheme of simply supported beams and with one of the first three natural frequencies close to $2 \mathrm{~Hz}$. The proposed method could be a very effective way to evaluate response of the structure to a single pedestrian load, because it does not require a time analysis and can be simply performed in commercial static software.

Parametric study in section 3 proved it is mainly length of the bridge and structural damping that influences the reduction coefficient. The numerical analysis in section 4 proved the estimation of reduction factor $\mathrm{R}=0.6-0.7[1$ for low damped structures. However, it also showed that these approximations can lead to underestimation of load, which was demonstrated on the structure with TMD in this case.

Verification of the proposed formula was performed with very accurate results. Nevertheless, further experimental verification and investigation in this field shall be performed.

\section{ACKNOWLEDGEMENTS}

The authors gratefully acknowledge support from the Czech Technical University in Prague, project SGS18/037/OHK1/1T/11 Development and application of advanced algorithms for numerical analysis and modeling in mechanics of structures and materials

\section{REFERENCES}

[1] Štimac Grandić. Serviceability verification of pedestrian bridges under pedestrian loading. Tehnički vjesnik 22:527-537, 2015. DOI:10.17559/TV-20131030105641

[2] SETRA. Footbridges: Assessment of vibrational behaviour of footbridges under pedestrian loading, 2006.

[3] FIB Bulletin 32: Guidelines for the design of footbridges, 2005.

[4] S. Živanović, A. Pavić, P. Reynolds. Vibration serviceability of footbridges under human-induced excitation: a literature review. Journal of Sound and Vibration 279(1-2):1-74, 2015. DOI:10.1016/j.jsv.2004.01.019.

[5] A. Dazio. Fundamentals of structural dynamics. An-Najah National University, 2013.

[6] Guide to basis of bridge design related to eurocodes supplemented by practical design - handbook 4 . Leonardo da Vinci pilot project, 2004.

[7] E. Caetano, A. Cunha, W. Hoorpah, J. Raoul. Footbridge Vibration Design. CRC Press, 2009.

[8] E. Shahabpoor, A. Pavic, V. Racic. Interaction between walking humans and structures in vertical direction: A literature review. Shock and vibration p. 22, 2016. DOI:10.1155/2016/3430285 